\title{
STUDY OF EXPONENTIAL STABILITY OF COUPLED WAVE SYSTEMS VIA DISTRIBUTED STABILIZER
}

\author{
MAHMOUD NAJAFI
}

(Received 23 August 1999)

\begin{abstract}
Stabilization of the system of wave equations coupled in parallel with coupling distributed springs and viscous dampers are under investigation due to different boundary conditions and wave propagation speeds. Numerical computations are attempted to confirm the theoretical results.
\end{abstract}

2000 Mathematics Subject Classification. 58J45, 93D15, 93D20, 37D99, 35L05.

1. Introduction. Many problems in structural dynamics deal with stabilizing the elastic energy of partial differential equations by boundary or internal energy dissipative controllers for wave equations or the Euler-Bernoulli beam equation. Exponential stability is a very desirable property for such elastic systems. The energy multiplier method [2, 6] has been successfully applied to reach to this objective for various partial differential equations and boundary conditions. Stabilization properties of serially connected vibrating strings or beams can be found in several papers [4, 5]. There, uniform stabilization can be achieved if we employ dissipative boundary condition at one end. If otherwise, one damper is located at the mid-span joint of two vibrating strings coupled in series, the uniform stabilization property holds if $c_{1} / c_{2}$ (wave speeds) has certain rational values. Stabilization properties of parallel connected vibrating strings were investigated under various end conditions by [9]. What comes new in this work is, firstly, dealing with the system of wave equations coupled in parallel with distributed viscous damping and springs (suspension system), and secondly, the rate of convergence of the solution when this system goes under the movement by an external disturbance (forcing function) or initial conditions. Having considered this, we are willing to furnish the best possible configuration that guarantees the uniform exponential stability due to different boundary conditions and wave speeds.

Let $\Omega_{1}=\Omega_{2}=\Omega=(0,1)$ be open sets in $\mathbb{R}$. Also, let $\partial \Omega_{1}, \partial \Omega_{2}$ be the boundaries of $\Omega_{1}$ and $\Omega_{2}$, respectively. Throughout, $(\cdot)=d() / d t,\left(^{\prime}\right)=d() / d x$, and $\partial_{x}^{2}()=\left(\partial^{2} / \partial x^{2}\right)()$. The coupling constants $\beta>0$ and $\alpha>0$ are damping and spring coefficients, respectively. We assume that the projection of $\Omega_{1}$ into $\Omega_{2}$, denoted as $\Omega$. Also, $u(x, t)$ and $v(x, t)$ are the displacement of two vibrating strings measured from their equilibrium positions.

The governing equations prescribing the above systems are

$$
\begin{array}{ll}
u_{t t}-c_{1}^{2} u_{x x}=\alpha(v-u)+\beta\left(v_{t}-u_{t}\right), & \text { in } \Omega_{1} \times(0, \infty), \\
v_{t t}-c_{2}^{2} v_{x x}=\alpha(u-v)+\beta\left(u_{t}-v_{t}\right), & \text { in } \Omega_{2} \times(0, \infty),
\end{array}
$$


with the initial conditions

$$
\begin{array}{lll}
u(0)=f_{1}, & u_{t}(0)=g_{1}, & \text { in } \Omega_{1}, \\
v(0)=f_{2}, & v_{t}(0)=g_{2}, & \text { in } \Omega_{2} .
\end{array}
$$

Along with the system (1.1), we employ two different boundary conditions and wish to study the stabilization of (1.1) on each, respectively. They are

(1) Dirichlet and Neumann for (1.1a) and Dirichlet for (1.1b):

$$
\begin{gathered}
u(0, t)=0, \quad u_{x}(1,0)=0, \quad \text { on } \partial \Omega_{1} \times(0, \infty), \\
v=0, \quad \text { on } \partial \Omega_{2} \times(0, \infty) .
\end{gathered}
$$

(2) Dirichlet for (1.1):

$$
u=v=0, \quad \text { on } \partial \Omega \times(0, \infty) .
$$

Here, $c_{1}$ and $c_{2}$ are wave speeds, also the distributed springs and dampers linking two vibrating strings are the coupling terms, that is, $\alpha(u-v)$ and $\beta\left(u_{t}-v_{t}\right)$. Energy can flow from one object to another through this parameter $(\alpha)$ and damp via shock absorber $(\beta)$. This system is well posed in the following sense: if we put

$V=\left\{\bar{X}=\left[u_{1}, v_{1}\right]^{T} ; u_{1} \in H^{1}\left(\Omega_{1}\right), v_{1} \in H^{1}\left(\Omega_{2}\right) \mid u_{1}=0\right.$, or $u_{1 x}=0$ on $\partial \Omega_{1}, v_{1}=0$ on $\left.\partial \Omega_{2}\right\}$,

for any initial data $\left(f_{1}, g_{1}, f_{2}, g_{2}\right) \in V \times L^{2}(\Omega)$, the system (1.1), (1.2), (1.3), (1.4), and (1.5) has a unique solution, satisfying

$$
\bar{X} \in C([0, \infty) ; V) \cap C^{1}\left([0, \infty) ; L^{2}(\Omega)\right) .
$$

Furthermore, the system is dissipative: the energy of the solutions, defined by

$$
E(t)=\frac{1}{2} \int_{0}^{1}\left\{\left|u_{t}\right|^{2}+c_{1}^{2}\left|\partial_{x} u\right|^{2}+\left|v_{t}\right|^{2}+c_{2}^{2}\left|\partial_{x} v\right|^{2}+\alpha|u-v|^{2}\right\} d x,
$$

is decreasing in $t \in(0, \infty)$, since

$$
\dot{E}=-\beta \int_{0}^{1}\left|u_{t}-v_{t}\right|^{2} d x \leq 0 .
$$

This paper consists of two main parts with their corresponding subsections. In the first part, we study the cases for boundary conditions (1.3) and (1.4), respectively. In Section 2, we set our notation and reformulate the system (1.1), (1.2), (1.3), and (1.4) into an evolution system and discuss more about well-posedness of the problem. There, we also give a set of sufficient conditions for exponential decay. The theorems formulated in terms of the influence of the bounded operator $B$ on the separated eigenmodes or clustered eigenmodes of $\mathscr{A}$ (system operator), see (2.4). Section 3 , which is the direct application of Theorem 2.2, in Section 2, we prove that the solution (1.1) with (1.3) decays to zero uniformly exponentially. In Section 4 , the importance of different wave speeds has to be taken into consideration for exponential stability of (1.1) with (1.4). To do this, we use the spectral method.

The second part, Section 5, of this paper is concerned with numerical computations. There, we study the behaviour of solutions of the system graphically (using finite 
different scheme [11]) as time increases for boundary condition (1.4) and show the significant stability of the solutions due to different wave propagation speeds.

2. Notations and preliminaries. Let the operator $A$ be defined as

$$
A u=-\partial_{x}^{2} u
$$

where $A: D(A) \rightarrow H^{0}(\Omega)=L^{2}(\Omega)$, and domain of $A, D(A)=\left\{u \in H^{2}(\Omega) ;\left.u\right|_{x \in \partial \Omega_{1}}=0\right\}$, and $H^{0}(\Omega)=L^{2}(\Omega)$ is endowed with the usual Hilbert space topology. Properties of the operator $A$ :

(1) Closed and densely defined operator.

(2) Selfadjoint and coercive.

(3) $A^{-1}$ is a compact operator.

Then, we can conclude that from the spectral theory of the selfadjoint operators with compact resolvent that

$$
\sigma(A)=\sigma_{p}(A)=\left\{\lambda_{n}^{2}\right\}_{1}^{\infty},
$$

where $\lambda_{n}>0, \lambda_{n} \rightarrow \infty$, and they are isolated, and each eigenvalue has a finite multiplicity. The corresponding normalized eigenvectors form an orthonormal basis for $H^{0}$. We also define a Hilbert space $H^{1}=D\left(A^{1 / 2}\right)$ endowed with inner product

$$
\left\langle v_{1}, v_{2}\right\rangle_{H^{1}}=\left\langle A^{1 / 2} v_{1}, A^{1 / 2} v_{2}\right\rangle_{H^{0}} .
$$

Now, the system (1.1) with boundary condition (1.4), similar approach can be applied for other boundary conditions, can be reformulated into a first order evolution system

$$
\frac{d}{d t} X(\cdot, t)=\mathscr{A} X(\cdot, t)+B X(\cdot, t), \quad X(\cdot, 0)=X_{0},
$$

in a Hilbert space $\mathscr{H}_{1}$. Here,

$$
\begin{gathered}
\mathscr{A}=\left[\begin{array}{cccc}
0 & 1 & 0 & 0 \\
-c_{1}^{2} A-\alpha & 0 & \alpha & 0 \\
0 & 0 & 0 & 1 \\
\alpha & 0 & -C_{2}^{2} A-\alpha & 0
\end{array}\right], \quad B=\left[\begin{array}{cccc}
0 & 0 & 0 & 0 \\
0 & -\beta & 0 & \beta \\
0 & 0 & 0 & 0 \\
0 & \beta & 0 & -\beta
\end{array}\right], \\
X(\cdot, t)=\left(u(\cdot, t), u_{t}(\cdot, t), v(\cdot, t), v_{t}(\cdot, t)\right)^{T} \in \mathscr{H}_{1}, \\
B X(\cdot, t)=\left(0,-\beta\left(u_{t}-v_{t}\right), 0, \beta\left(u_{t}-v_{t}\right)\right)^{T},
\end{gathered}
$$

with

$$
\mathscr{H}_{2}=D(\mathscr{A})=D(A) \times H^{1} \times D(A) \times H^{1} \subset \mathscr{H}_{1} .
$$

The Hilbert space $\mathscr{H}_{1}=H^{1} \times H^{0} \times H^{1} \times H^{0}$ is endowed with inner product

$$
\begin{aligned}
\left\langle u_{1}, u_{2}\right\rangle_{\mathscr{H}_{1}}= & c_{1}^{2}\left\langle u_{1}, u_{2}\right\rangle_{H^{1}}+\left\langle z_{1}, z_{2}\right\rangle_{H^{0}}+c_{2}^{2}\left\langle v_{1}, v_{2}\right\rangle_{H^{1}} \\
& +\left\langle w_{1}, w_{2}\right\rangle_{H^{0}}+\alpha\left\langle\left(u_{1}-v_{1}\right),\left(u_{2}-v_{2}\right)\right\rangle_{H^{0}},
\end{aligned}
$$

where $u_{1}=\left(u_{1}, z_{1}, v_{1}, w_{1}\right)^{T}$ and $u_{2}=\left(u_{2}, z_{2}, v_{2}, w_{2}\right)^{T}$. It can be shown that $\mathscr{H}_{2}$ is dense in $\mathscr{H}_{1}$. 
Energy of the system (1.7) can be rewritten as

$$
E(t)=\frac{1}{2} \int_{0}^{1}\left\{\left|u_{t}\right|^{2}+c_{1}^{2}\left|A^{1 / 2} u\right|^{2}+\left|v_{t}\right|^{2}+c_{2}^{2}\left|A^{1 / 2} v\right|^{2}+\alpha|u-v|^{2}\right\} d x .
$$

It is known that the unbounded operator $\mathscr{A}$ is closed and densely defined from $D(\mathscr{A})$ to $\mathscr{H}_{1}$. Furthermore, $\mathscr{A}$ has the following properties [10].

LEMMA 2.1. Let $\mathscr{A}: D(\mathscr{A}) \rightarrow \mathscr{H}_{1}$, and closed and densely defined operator, then

(a) $\mathscr{A}=-\mathscr{A}^{*}$ (skew-adjoint),

(b) A has compact resolvent $\mathscr{A}^{-1}$,

(c) $A$ is the infinitesimal generator of a $C_{0}$-semigroup $\hat{S}(t)$.

Proof. (a) For any $u_{1} \in D(\mathscr{A})$ and $u_{2} \in D(-\mathscr{A})=D(\mathscr{A})$, we have

$$
\begin{aligned}
\left\langle A \mathcal{A} u_{1}, u_{2}\right\rangle_{H_{1}}= & c_{1}^{2}\left\langle z_{1}, u_{2}\right\rangle_{H^{1}}+\left\langle-\left(c_{1}^{2} A+\alpha\right) u_{1}+\alpha v_{1}, z_{2}\right\rangle_{H^{0}}+c_{2}^{2}\left\langle w_{1}, v_{2}\right\rangle_{H^{1}} \\
& +\left\langle\alpha u_{1}-\left(c_{2}^{2} A+\alpha\right) v_{1}, w_{2}\right\rangle_{H^{0}}+\left\langle\alpha\left(u_{1}-v_{1}\right),\left(u_{2}-v_{2}\right)\right\rangle_{H^{0}} \\
= & -c_{1}^{2}\left\langle u_{1}, z_{2}\right\rangle_{H^{1}}+\left\langle z_{1}, c_{1}^{2} A u_{2}\right\rangle_{H^{0}}+\alpha\left\langle\left(v_{1}-u_{1}\right), z_{2}\right\rangle_{H^{0}} \\
& +\left\langle w_{1}, c_{2}^{2} A v_{2}\right\rangle_{H^{0}}+\alpha\left\langle\left(u_{1}-v_{1}\right), w_{2}\right\rangle_{H^{0}}-c_{2}^{2}\left\langle v_{1}, w_{2}\right\rangle_{H^{1}} \\
& +\alpha\left\langle z_{1},\left(u_{2}-v_{2}\right)\right\rangle_{H^{0}}+\alpha\left\langle w_{1},\left(u_{2}-v_{2}\right)\right\rangle_{H^{0}} \\
= & \left\langle u_{1},-\mathscr{A} u_{2}\right\rangle_{\mathscr{H}_{1}} .
\end{aligned}
$$

This implies that $D(-\mathscr{A}) \subset D\left(\mathscr{A}^{*}\right)$ and $\left.\mathscr{A}^{*}\right|_{D(-\mathscr{A})}=-\mathscr{A}$. In [7], we can verify that $D\left(\mathscr{A}^{*}\right) \subset D(-\mathscr{A})=D(A)$. Hence, $D(\mathscr{A})=D\left(-\mathscr{A}^{*}\right)$.

(b) The compactness of $\mathscr{A}^{-1}$ follows from the Sobolev embedding theorem.

(c) Since $\mathscr{A}$ is a closed linear operator with dense domain in $\mathscr{H}_{1}$, and

$$
\begin{array}{cc}
\langle\mathscr{A} X, X\rangle_{\mathscr{H}_{1}}+\langle X, \mathscr{A} X\rangle_{\mathscr{H}_{1}}=0 & \forall X \in D(\mathscr{A}), \\
\left\langle\mathscr{A}^{*} X, X\right\rangle_{\mathscr{H}_{1}}+\left\langle X, \mathscr{A}^{*} X\right\rangle_{\mathscr{H}_{1}}=0 & \forall X \in D(\mathscr{A}),
\end{array}
$$

then, by [1, Corollary 4.3.1], $A$ generates $C_{0}$-semigroup that preserves norms, and that ends to the proof of Lemma 2.1.

Now, let $\tilde{A}=\mathscr{A}+B$ with domain $D(\tilde{A})=D(\mathscr{A}) \subset \mathscr{H}_{1}$. By [10, Theorem 1.1], since $B$ is a bounded linear operator in $\mathscr{H}_{1}$, and by Lemma 2.1, we can pose the following theorem which furnishes the well-posedness of the problem.

THEOREM 2.2. The operator $\tilde{A}$ is the infinitesimal generator of a $C_{0}$-semigroup $\tilde{S}(t)$.

Equation (2.4) without operator $B$ is said to be energy-conserving if it satisfies

$$
\left\|\hat{S}(t) X_{0}\right\|=\left\|X_{0}\right\|, \quad \forall X_{0} \in \mathscr{H}_{1}, t \geq 0 .
$$

Wave propagation, quantum phenomenon, and mechanical vibration are the examples of this type. Usually energy dissipation comes into account when there exists a medium impurity, distributed on boundary frictions, small viscous effects, and so forth. These factors cannot be ignored, therefore we must incorporate them into (2.4). Hence, $B X(\cdot, t)$ is the perturbing term which satisfies energy dissipation which causes 
the energy of the system to decay, that is, in structural dynamics, viscous damping material suppresses the vibration. Hence, (2.4) with $B$ describes stabilization problems.

From Theorem 2.2, $\tilde{A}$ is a dissipative operator and that generates a $C_{0}$-semigroup of contractions $\tilde{S}(t)$ :

$$
\|\tilde{S}(t)\| \leq 1, \quad \forall t \geq 0 .
$$

Now, the question is: does the uniformly exponentially decay happen? That is, there are $\omega>0, K \geq 1$ such that

$$
\|\tilde{S}(t)\| \leq K e^{-\omega t} .
$$

This is a stabilization problem and implies that all the eigenmodes should be damped out uniformly at some rate $\omega$. We end this section with pointing out the following assumptions which are the sufficient conditions for exponential decay [3]. These assumptions are formulated in terms of the influence of the operator $B$ on the separated eigenmodes or clustered eigenmodes of $\mathscr{A}$.

Suppose in (2.4) we have

(A1) $\mathscr{A}^{*}=-\mathscr{A}$.

(A2) $\mathscr{A}$ has a compact resolvent $R(\lambda ; \mathscr{A})=(\lambda I-\mathscr{A})^{-1}$ for some $\lambda \in \mathbb{C}$ (hence for all $\lambda$ in the resolvent set of $\mathscr{A})$, that is, $\operatorname{Ker} \mathscr{A}=\{0\}$.

(A1) and (A2) imply that $\mathscr{A}$ has a complete orthonormal set of eigenfunctions (eigenvectors) with corresponding eigenvalues $i \lambda$ 's.

(A3) The spectrum of $\mathscr{A}$ satisfies the gap property

$$
\inf \left\{\left|\lambda_{j}-\lambda_{k}\right|: j, k=1,2,3, \ldots, j \neq k\right\}=\gamma>0 .
$$

From (2.14) we can conclude that the spectrum of $\mathscr{A}$ in one space dimension, under the compact resolvent condition (A2), consists of a discrete spectrum which is separated by a steady gap, that is, $\gamma$.

(A4) The bounded linear operator $B$ is dissipative:

$$
\operatorname{Re}\langle B X, X\rangle \leq 0, \quad \forall X \in \mathscr{H}_{1} .
$$

(A1) and (A4) lead to the dissipativeness of operator $\tilde{A}$. These assumptions along with (A3) imply that $\tilde{A}$ has compact resolvents by the following corollary [7].

COROLlary 2.3. Let $(\mathscr{A})$ be skew-adjoint and $\mathscr{A}^{-1}$ be compact. Let $B$ be a bounded linear operator, then there exists at least $\lambda \in \mathbb{C}$ which is not an eigenvalue of $\tilde{A}$, and $(\tilde{A}-\lambda I)^{-1}$ exists and is compact.

Note that by Lumer-Phillips theorem [10], Ã generates a $C_{0}$-contraction semigroup $\tilde{S}(t)=e^{\tilde{A} t}$ (also by Theorem 2.2).

(A5) If any sequence $\left\{x_{n} \in \mathscr{H}_{1}: n=1,2, \ldots\right\}$ satisfies

$$
\lim _{n \rightarrow \infty} \operatorname{Re}\left\langle B x_{n}, x_{n}\right\rangle=0,
$$

then

$$
\lim _{n \rightarrow \infty} B x_{n}=0 .
$$


(A6) There exists $\delta>0$ such that $\|B \psi\| \geq \delta$ for any unit eigenvector $\psi$ of $\mathscr{A}$; that is,

$$
\|\psi\|=1, \quad \mathscr{A} \psi=i \lambda_{n} \psi \text { for some } n
$$

Note that (A1)-(A3) deal with the operator $\mathscr{A}$ which is unperturbed. And, (A1)-(A6) are concerned only with damping perturbation operator $B$.

MAIN THEOREM 2.4 (see [3]). Under assumptions (A1)-(A6) property (2.13) holds.

3. Exponential stability (frequency domain method). Uniform exponential decay of system (1.1) with (1.3) in $\mathbb{R}^{1}$, which is the main course of this paper, is under consideration. To do this, we pose the following theorem.

THEOREM 3.1. Let $\Omega=\{x \mid x \in(0,1)\}$ and $\alpha(x)$ and $\beta(x)>\beta_{0}$ be positive qualities, bounded and continuous over the subinterval $I \subset(0,1)$, then the solution of the following system:

$$
\begin{array}{ll}
u_{t t}-c_{1}^{2} u_{x x}=\alpha(v-u)+\beta\left(v_{t}-u_{t}\right), & 0<x<1, \\
v_{t t}-c_{1}^{2} v_{x x}=\alpha(u-v)+\beta\left(u_{t}-v_{t}\right), & 0<x<1,
\end{array}
$$

with the initial conditions

$$
u(0)=u^{0}, \quad u_{t}(0)=u^{1}, \quad v(0)=v^{0}, \quad v_{t}(0)=v^{1},
$$

along with boundary conditions

$$
u(0, t)=0, \quad u_{x}(1, t)=0, \quad v(0, t)=0, \quad v(1, t)=0, \quad t>0
$$

will be uniformly exponential decay.

Proof. This theorem is the direct application of Theorem 2.4. The underlying Hilbert space is

$$
\mathscr{H}_{1}=\left\{U_{1} \in H^{1}(0,1) \times L^{2}(0,1) \times H^{1}(0,1) \times L^{2}(0,1) \mid u_{0}(0)=v_{0}(0)=0\right\},
$$

where $H^{m}(0,1)$ is the standard Sobolev space of order $m$. The inner product is defined by

$$
\left\langle U_{1}, U_{2}\right\rangle_{\mathscr{H}_{1}}=\int_{0}^{1}\left\{c_{1}^{2} u_{0}^{\prime} \bar{w}_{0}^{\prime}+u_{1} \bar{w}_{1}+c_{2}^{2} v_{0}^{\prime} \bar{z}_{1}^{\prime}+v_{1} \bar{z}_{1}+\alpha\left(u_{0}-v_{0}\right)\left(\bar{w}_{0}-\overline{z_{0}}\right)\right\} d x,
$$

where

$$
U_{1}=\left(u_{0}, u_{1}, v_{0}, v_{1}\right)^{T}, \quad U_{2}=\left(w_{0}, w_{1}, z_{0}, z_{1}\right)^{T} .
$$

Without loss of generality, assume that $c_{1}=c_{2}=1$, and $\alpha=\beta=1$. Define (see [9])

$$
\hat{A}=\left[\begin{array}{cccc}
0 & 1 & 0 & 0 \\
\partial_{x}^{2}-1 & 0 & 1 & 0 \\
0 & 0 & 0 & 1 \\
1 & 0 & \partial_{x}^{2}-1 & 0
\end{array}\right], \quad B=\left[\begin{array}{cccc}
0 & 0 & 0 & 0 \\
0 & -1 & 0 & 1 \\
0 & 0 & 0 & 0 \\
0 & 1 & 0 & -1
\end{array}\right]
$$


Then $\hat{A}$ has a complete orthonormal set of eigenfunctions:

$$
\Phi_{n}=\frac{1}{\left\|\Phi_{n}\right\|_{\mathscr{H}_{1}}}\left(u_{0}, i \lambda u_{0}, v_{0}, i \lambda v_{0}\right)^{T},
$$

where in [9], we have found

$$
\begin{aligned}
& u_{0}=\left(\frac{\sin a x}{a}+\frac{\sin b x}{b}\right) K_{2}+\left(\frac{\sin a x}{a}-\frac{\sin b x}{b}\right) K_{4}, \\
& v_{0}=\left(\frac{\sin a x}{a}-\frac{\sin b x}{b}\right) K_{2}+\left(\frac{\sin a x}{a}+\frac{\sin b x}{b}\right) K_{4} .
\end{aligned}
$$

To find corresponding eigenvalues, we should apply the right end boundary conditions (3.3). One can get the following homogeneous equations:

$$
\left[\begin{array}{cc}
\cos a+\cos b & \cos a-\cos b \\
\frac{\sin a}{a}-\frac{\sin b}{b} & \frac{\sin a}{a}+\frac{\sin b}{b}
\end{array}\right]\left[\begin{array}{l}
K_{2} \\
K_{4}
\end{array}\right]=0 .
$$

The homogeneous system (3.10) can give nonzero values for the unknown coefficients $K_{i}, i=2,4$, only provided that the determinant of the matrix on the left side is zero. This leads to the following condition:

$$
h \cos a \sin b+\cos b \sin a=0,
$$

where

$$
a=\lambda, \quad b=\sqrt{\lambda^{2}-2}, \quad h=\frac{a}{b} .
$$

Equation (3.11) is the frequency equation, and will lead to an infinite number of values for $\lambda$ (eigenvalues). Corresponding to each value of $\lambda$, a solution can be obtained for eigenfunction, by substituting for $\lambda$ in (3.10), solving those equations for coefficients $K_{i}, i=2,4$, and substituting the resulting values of $K_{i}, i=2,4$, in (3.9). So, we have from (3.10)

$$
K_{2}=\tan \frac{1}{2}(a+b) \tan \frac{1}{2}(a-b) K_{4}
$$

Substitute (3.13) into (3.9) for $K_{2}$, one can obtain the following eigenfunctions defined in (3.8):

$$
\begin{aligned}
& u_{0}=K_{4}\left\{\tan \frac{1}{2}(a+b) \tan \frac{1}{2}(a-b)\left(\frac{\sin a x}{a}+\frac{\sin b x}{b}\right)+\left(\frac{\sin a x}{a}+\frac{\sin b x}{b}\right)\right\}, \\
& v_{0}=K_{4}\left\{\tan \frac{1}{2}(a+b) \tan \frac{1}{2}(a-b)\left(\frac{\sin a x}{a}-\frac{\sin b x}{b}\right)+\left(\frac{\sin a x}{a}+\frac{\sin b x}{b}\right)\right\} .
\end{aligned}
$$

The COMPLETION PROOF OF THEOREM 3.1. Now, we utilize the assumptions of Theorem 2.4.

(A1) and (A2) are satisfied by the Lemma 2.1. (A3) (gap properties) can be reached from (3.10). (A4) the bounded linear operator $B$ is dissipative, since

$$
\operatorname{Re}\left\langle B U_{1}, U_{1}\right\rangle=-\int_{0}^{1} \beta(x)\left|u_{1}-v_{1}\right|^{2} d x \leq 0,
$$

where from [9], we have

$$
\begin{aligned}
& u_{1}=(\cos a x+\cos b x) K_{2}+(\cos a x-\cos b x) K_{4}, \\
& v_{1}=(\cos a x-\cos b x) K_{2}+(\cos a x+\cos b x) K_{4} .
\end{aligned}
$$

(A5) can be verified easily. 
(A6) There exists $\delta>0$ such that $\|B \psi\| \geq \delta$ for any unit eigenvector $\psi$ of $\hat{A}$ (i.e., $\|\psi\|=1, A \psi=i \lambda_{n} \psi$, for some $n$ ). From (3.7), (3.8), and (3.9), we have

$$
\left\|B \Phi_{n}\right\|_{\mathscr{H}_{1}}^{2}=\left\langle B \Phi_{n}, B \Phi_{n}\right\rangle_{\mathscr{H}_{1}}=\frac{8 \lambda_{n}^{2}}{b^{2}}\left(K_{2}-K_{4}\right)^{2} \int_{0}^{1} \beta(x)^{2} \sin ^{2}(b x) d x .
$$

Equation (3.13) raises a very serious question: what are the values of $K_{2}$ and $K_{4}$ for large $n$ ? Because, if $K_{2}=K_{4}$, according to the following corollary, we do not have the uniform exponential decay for (1.1).

COROLlary 3.2 (see [3]). Let Â and B satisfy the assumptions (A1)-(A5). In addition, suppose that there exists a sequence of unit eigenvectors of $\hat{A},\left\{\psi_{n} \mid n=1,2, \ldots\right\}$, each member of which corresponds to an eigenvalue $i \lambda_{n}$, such that $\left\|B \psi_{n}\right\| \rightarrow 0$ as $n \rightarrow \infty$. Then the uniform exponential decay property (2.13) fails.

To answer this question, we should look back to (3.17) and find out the behaviour of $K_{2}$ and $K_{4}$ for large $\lambda$. To do this, substitute (3.13) into (3.17) for $K_{2}$, and let $\lambda \rightarrow \infty$. Finally, we can derive $\left\|B \Phi_{n}\right\|_{\mathscr{H}_{1}}^{2} \geq 4 \beta_{0}^{2} K_{4}^{2}=\delta^{2}>0$, which leads us to the completion of the proof of Theorem 3.1.

4. Strongly decay (spectral method). Separation of variables is extremely valuable when there are time derivatives. It is also extremely direct, because the part involving time is only an exponential. For the heat equation it is a decay $e^{-\lambda t}$, and for the wave equation it is an oscillation $e^{-i \omega t}$. The key is to find the eigenvectors. They solve the time-dependent problem by combining with $e^{-\lambda t}$ or $e^{i \omega t}$ into pure exponential solutions. For partial differential equations they are eigenfunctions. That is the step from matrices to derivatives, which takes us directly to the fundamental equation (1.1). The terms, $\partial^{2}(\cdot) / \partial x^{2}$ has negative eigenvalues $\left(\partial^{2} / \partial x^{2}\right)\left(e^{2 \pi i k x}\right)=-(2 \pi k)^{2} e^{2 \pi i k x}$ for a periodic case, and $\left(\partial^{2} / \partial x^{2}\right)(\sin \pi k x)=-(\pi k)^{2} \sin (\pi k x)$ for zero boundary conditions. The separated solutions $a(t) \phi(x)$ can be written down immediately. The heat equation has decaying solutions $e^{-\lambda t} \phi$; the wave equation has oscillating solutions $e^{i \omega t} \phi$ and $e^{-i \omega t} \phi$. The eigenvalues $-\lambda=-\omega^{2}$ with the eigenfunctions $\phi$-one for each frequency $k$. The solutions to wave equations are combinations of these exponential solutions. For example,

$$
u=\sum\left(c_{k} e^{i \omega_{k} t}+d_{k} e^{-i \omega_{k} t}\right) \phi_{k}(x) .
$$

To solve (1.3) with (1.4) we assume that the solutions can be written as a sum of complete sets of eigenfunctions on $0 \leq x \leq 1$, namely

$$
u=\sum a_{k}(t) \sin k \pi x, \quad v=\sum b_{k}(t) \sin k \pi x .
$$

What is unique about this approach is that it may be generalized so that any infinite series of smooth and, preferably, orthogonal functions may be used to eliminate the physical space variable from the problem and reduce the solutions of the partial differential equations to the solution of a set of ordinary differential equations in the other independent variable, that is, time. Because of its close association with the Fourier series, the expansion coefficients are referred to as a spectra and this approach is called the spectral method. Now, we introduce (4.2) into (3.1) for $u$ and $v$. One can get the following system of the linear, second-order, homogeneous ordinary 
differential equations in $a(t)$ and $b(t)$ :

$$
\begin{aligned}
& \ddot{a}_{k}=-\left(c_{1} \pi k\right)^{2} a_{k}+\alpha\left(b_{k}-a_{k}\right)+\beta\left(\dot{b}_{k}-\dot{a}_{k}\right), \\
& \ddot{b}_{k}=-\left(c_{2} \pi k\right)^{2} b_{k}+\alpha\left(a_{k}-b_{k}\right)+\beta\left(\dot{b}_{k}-\dot{a}_{k}\right) .
\end{aligned}
$$

A general solution of (4.3) depends upon the magnitude of $k$. System (4.3) can be written as system of a first-order ordinary differential equations:

$$
\dot{\chi}=\bar{A} \chi,
$$

where $\chi=(a, \dot{a}, b, \dot{b})^{T}$, and let

$$
\Pi_{1}=-\left[\left(c_{1} \pi K\right)^{2}+\alpha\right], \quad \Pi_{2}=-\left[\left(c_{2} \pi K\right)^{2}+\alpha\right],
$$

then

$$
\bar{A}=\left[\begin{array}{cccc}
0 & 1 & 0 & 0 \\
\Pi_{1} & -\beta & \alpha & \beta \\
0 & 0 & 0 & 1 \\
\alpha & \beta & \Pi_{2} & -\beta
\end{array}\right] .
$$

The characteristic polynomial of the matrix $\bar{A}$ is

$$
P(\lambda)=\operatorname{det}(\bar{A}-\lambda I)=\lambda^{4}+2 \lambda^{3}+\left(a_{1}+a_{2}+2\right) \lambda^{2}+\left(a_{1}+a_{2}\right) \lambda+a_{1} a_{2}+a_{1}+a_{2},
$$

where $a_{1}=\left(c_{1} \pi K\right)^{2}$, and $a_{2}=\left(c_{2} \pi K\right)^{2}$. Here, $\alpha=\beta=1$. Solve (4.7) for $\lambda$ and study the behaviour of eigenvalues $(\lambda)$ for large $K$. Now, we pose the following theorem which is the essence of this section.

THEOREM 4.1. System (1.1) with (1.4) (i) is not uniformly stable if $c_{1}=c_{2}$, and (ii) is strongly stable if $c_{1} \neq c_{2}$.

Proof of (i). Consider the characteristic polynomial (4.7), and let the wave speeds be $c_{1}=c_{2}=1$ and $a=a_{1}=a_{2}$, then one can have

$$
\lambda^{4}+2 \lambda^{3}+2(a+1) \lambda^{2}+2 a \lambda+a^{2}+2 a=0 .
$$

Now, we produce the Roth's tabulation (see the appendix)

$$
\begin{array}{cccc}
\lambda^{4} & 1 & 2(a+1) & a^{2}+2 a \\
\lambda^{3} & 2 & 2 a & 0 \\
\lambda^{2} & a+2 & a^{2}+2 a & \\
\lambda^{1} & 0 & 0 &
\end{array}
$$

Since a row of zeros appears, we form the auxiliary equation using the coefficients of the $\lambda^{2}$ row. The auxiliary equation is

$$
F(\lambda)=(a+2) \lambda^{2}+a^{2}+2 a=\lambda^{2}+a=0,
$$

from which

$$
\frac{d F(\lambda)}{d \lambda}=2 \lambda=0
$$


from which the coefficients 2 and 0 replace the zeros in the $\lambda^{1}$ row of the original tabulation. The remaining portion of the Roth's tabulation is

$$
\begin{array}{ccc}
\lambda^{1} & 2 & 0 \\
\lambda^{0} & a^{2}+2 a &
\end{array}
$$

Since there are no sign changes in the first column of Roth's tabulation, equation (4.8) does not have any root in the right-half complex-plane. Solving (4.10), we find $\lambda= \pm i a^{1 / 2}= \pm i(c \pi K)$, which are also the roots of (4.10). Since the equation has roots on the $i \omega$-axis (imaginary axis) for each $K$, the system is not uniformly stable.

Proof OF (ii). We are trying to show the asymptotic behavior of the solutions when $c_{1} \neq c_{2}$. Consider the characteristic (4.7),

$$
P(\lambda)=\lambda^{4}+2 \lambda^{3}+\left(a_{1}+a_{2}+2\right) \lambda^{2}+\left(a_{1}+a_{2}\right) \lambda+a_{1} a_{2}+a_{1}+a_{2} .
$$

Equation (4.13) can be factored to

$$
P(\lambda)=\left(\lambda^{2}+x \lambda+a_{1}-z+1\right)\left(\lambda^{2}+y \lambda+a_{2}+z+1\right),
$$

in which $x, y$, and $z$ can be found from the following system of equations:

$$
\begin{gathered}
x+y=2, \quad x\left(a_{1}-z+1\right)+y\left(a_{2}+z+1\right)=a_{1}+a_{2}, \\
\left(a_{1}-z+1\right)\left(a_{2}+z+1\right)=a_{1} a_{2}+a_{1}+a_{2} .
\end{gathered}
$$

Solve system (4.15) for $z, x, y$, one can find the following solution set:

$$
\left\{z=\frac{1}{2}\left[\left(a_{1}-a_{2}\right) \pm\left(\left(a_{1}-a_{2}\right)^{2}+4\right)^{1 / 2}\right], x=1+\frac{2}{a_{1}-a_{2}-2 z}, y=2-x\right\} .
$$

We see that every eigenvalue of the matrix $\bar{A}$ has $-1 / 2$ as real part, and

$$
\lim _{K \rightarrow \infty} x=1 .
$$

Thus, $x \simeq y \simeq 1$ as $K \rightarrow \infty$. This implies that if $c_{1} \neq c_{2}$, we could find a sequence of solutions to the system (1.1) with boundary condition (1.4) which approaches equilibrium state strongly as time increases. This implies that the system is strongly stable.

5. Numerical confirmation. Having considered Theorem 4.1, we solve characteristic equation (4.7) in order to study the behavior of eigenvalues of the system (1.1) with (1.4) for large $\lambda$. Figures 5.1(a), 5.1(b), 5.1(c), and 5.1(d) in Part A show, when $c_{1} \neq c_{2}$, that as $\lambda \rightarrow \infty$, the real part of complex roots of (4.14) approaches $-1 / 2$ (see Figure 5.1(a)). Consequently, the system is asymptotically stable; that is, the energy of the system, equation (1.7), goes to zero (see Figure 5.1(d)), and the displacements $u$ and $v$ approach equilibrium state as $t \rightarrow \infty$. To observe this, we solved system (3.1), for $\alpha=\beta=1$, numerically using finite difference method [11] and the solutions are plotted in Figures 5.1(b) and 5.1(c) for $u$ and $v$, respectively. In Figures 5.1(e), 5.1(f), 5.1(g), and 5.1(h) Part B, when $c_{1}=c_{2}$, the sequence of eigenvalues are approaching -1 and 0 for all values of $k$ (see Figure 5.1(e)). This implies that the energy of the system never gets settled since the system is conservative (see Figure 5.1(h)). Consequently, the solutions $u$ and $v$ do not go to the state of rest (see Figures 5.1(f) and 5.1(g)). 


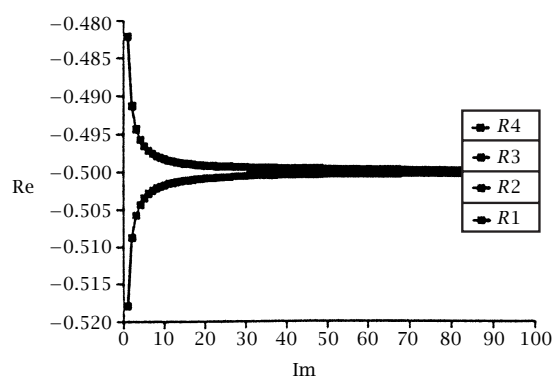

(a)

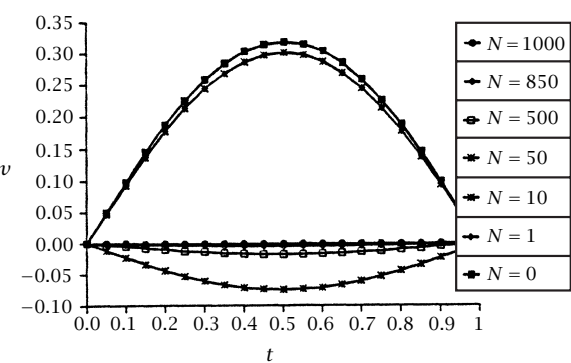

(c)

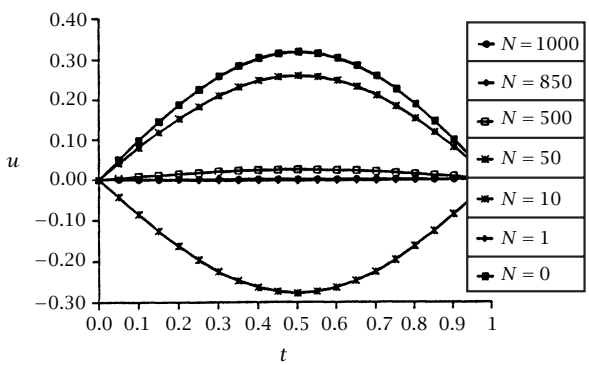

(b)

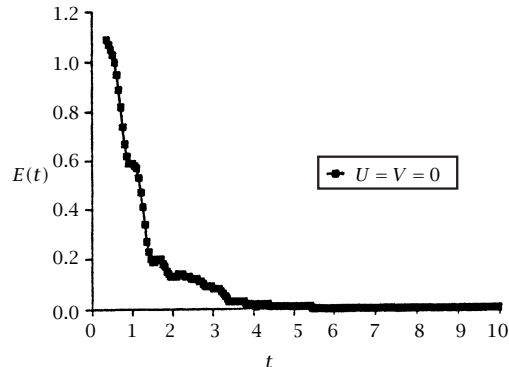

(d)

(A) $c_{1} \neq c_{2}$.

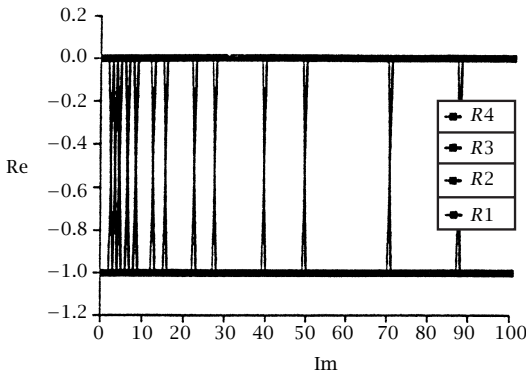

(e)

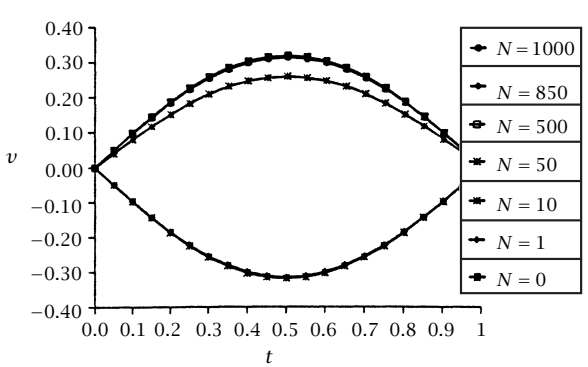

(g)

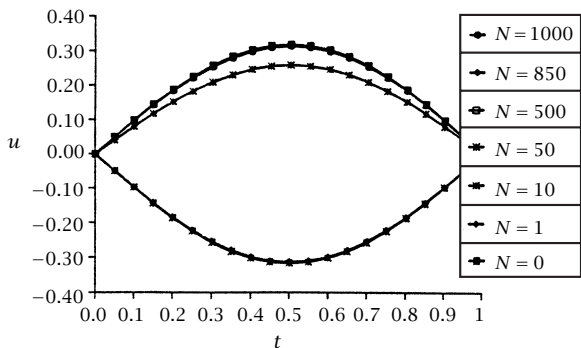

(f)

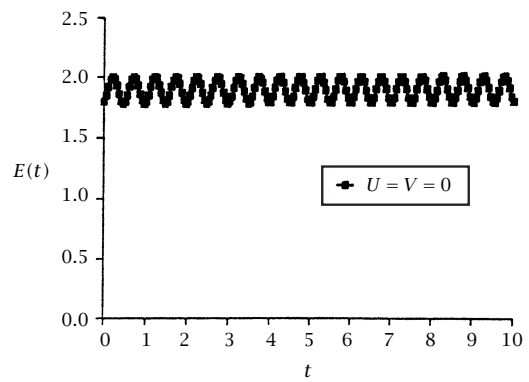

(h)

(B) $c_{1}=c_{2}$.

FIGURE 5.1. Numerical solutions to the system (1.1) with Dirichlet boundary conditions (1.4). 


\section{Appendix}

A.1. Routh-Hurwitz criterion. The Routh-Hurwitz criterion represents a method of determining the location of zeros of a polynomial with constant real coefficient with respect to the left-half and the right-half of the complex-plane, without actually solving for the zeros. Consider the following characteristic equation (with all real coefficients) of a linear time-invariant system

$$
P(\lambda)=a_{0} \lambda^{n}+a_{1} \lambda^{n-1}+a_{2} \lambda^{n-2}+\cdots+a_{n-1} \lambda+a_{n}=0 .
$$

In order that (A.1) does not have roots with positive real parts, it is necessary that the following conditions holds:

(i) All the coefficient of the (A.1) have the same sign.

(ii) None of the coefficients vanishes.

The above requirements are based on the laws of algebra, which relate the coefficient of (A.1).

A.2. The Hurwitz criterion. See [8].

TheOREM A.1 (Hurwits determinants). The necessary and sufficient condition that all roots of (A.1) lie in the left-half of the complex-plane is that the equation's Hurwitz determinants, $D_{k} k=1,2, \ldots, n$, must be positive. The Hurwitz determinants of (A.1) are given by

$$
\begin{aligned}
D_{1}=a_{1}, \quad D_{2} & =\left[\begin{array}{ll}
a_{1} & a_{3} \\
a_{0} & a_{2}
\end{array}\right], \quad D_{3}=\left[\begin{array}{ccc}
a_{1} & a_{3} & a_{5} \\
a_{0} & a_{2} & a_{4} \\
0 & a_{1} & a_{3}
\end{array}\right], \\
D_{n} & =\left[\begin{array}{cccc}
a_{1} & a_{3} & \cdots & a_{2 n-1} \\
a_{0} & a_{2} & \cdots & a_{2 n-2} \\
0 & a_{1} & \cdots & a_{2 n-3} \\
0 & 0 & 0 & a_{n}
\end{array}\right],
\end{aligned}
$$

where the coefficients with indices larger than $n$ or with negative indices are replaced with zeros. Routh simplified the process by introducing a tabulation method in place of the Hurwitz determinants, see proof of Theorem 4.1(i) and also in [8].

A.3. Special case. The following difficulty may occur that prevent Routh's tabulation from completing properly.

The elements in one row of Routh's tabulation are all zero. In this situation, one can use the auxiliary equation $F(\lambda)=0$. This equation is always an even polynomial, and the roots are also the roots of the original equation. In order to continue Routh's tabulation when this case happen, the following steps are needed:

(S1) From $F(\lambda)=0$ by use of the coefficients from the row one before the row of zeros.

(S2) Set $d F(\lambda) / d \lambda=0$ (the derivative $F(\lambda)$ with respect to $\lambda$ ).

(S3) Replace the row of zeros with the coefficient of $d F(\lambda) / d \lambda=0$.

(S4) Continue with Routh's tabulation in the usual manner with this row. 
(S5) Check the signs of the first column of Routh's tabulation which contains information on the roots of the equation. The roots of the equation are all in the half of the complex-plane if all the elements of this column are of the same sign.

\section{REFERENCES}

[1] A. V. Balakrishnan, Applied Functional Analysis, Applications of Mathematics, no. 3, Springer-Verlag, New York, 1976. MR 57\#10445. Zbl 333.93051.

[2] G. Chen, Energy decay estimates and exact boundary value controllability for the wave equation in a bounded domain, J. Math. Pures Appl. (9) 58 (1979), no. 3, 249-273. MR 81k:35093. Zbl 414.35044.

[3] G. Chen, S. A. Fulling, F. J. Narcowich, and S. Sun, Exponential decay of energy of evolution equations with locally distributed damping, SIAM J. Appl. Math. 51 (1991), no. 1, 266-301. MR 91k:35037. Zbl 734.35009.

[4] G. Chen, S. G. Krantz, D. L. Russell, C. E. Wayne, H. H. West, and M. P. Coleman, Analysis, designs, and behavior of dissipative joints for coupled beams, SIAM J. Appl. Math. 49 (1989), no. 6, 1665-1693. MR 90j:73055. Zbl 685.73046.

[5] G. Chen and H. Wang, Pointwise stabilization for coupled quasilinear and linear wave equations, Proceeding of the 3rd International Conference of Distributed Parameter Systems, Vorau, Austria, 1986, Lecture Notes in Control and Information Sciences, vol. 102, 1987, pp. 40-63. Zbl 642.93048.

[6] J. Lagnese, Decay of solutions of wave equations in a bounded region with boundary dissipation, J. Differential Equations 50 (1983), no. 2, 163-182. MR 85f:35025. Zbl 536.35043.

[7] W. Littman, L. Markus, and Y. You, A note on stabilization and controllability of a hybrid elastic system with boundary control, Mathematical report, University of Minnesota, 1987.

[8] R. K. Miller and A. N. Michel, Ordinary Differential Equations, Academic Press, New York, 1982. MR 83k:34001. Zbl 552.34001.

[9] M. Najafi, G. R. Sarhangi, and H. Wang, Stabilizability of coupled wave equations in parallel under various boundary conditions, IEEE Trans. Automat. Control 42 (1997), no. 9, 1308-1312. MR 98d:93078. Zbl 883.93044.

[10] A. Pazy, Semigroups of Linear Operators and Applications to Partial Differential Equations, Applied Mathematical Sciences, vol. 44, Springer-Verlag, New York, 1983. MR 85g:47061. Zbl 516.47023.

[11] R. D. Richtmyer and K. W. Morton, Difference Methods for Initial-Value Problems, Interscience Tracts in Pure and Applied Mathematics, no. 4, John Wiley and Sons, New York, 1967. MR 36\#3515. Zbl 155.47502.

Mahmoud najafi: Department of Mathematics, Kent State University, 3325 West

13TH STREET, ASHTABUla, OH 44004, USA

E-mail address: najafi@ashtabu1a.kent.edu 


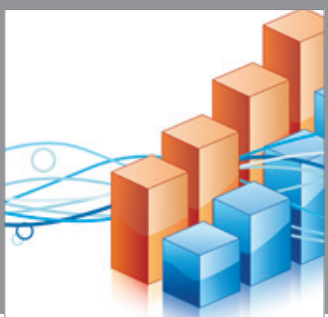

Advances in

Operations Research

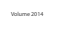

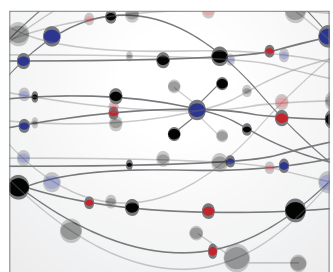

\section{The Scientific} World Journal
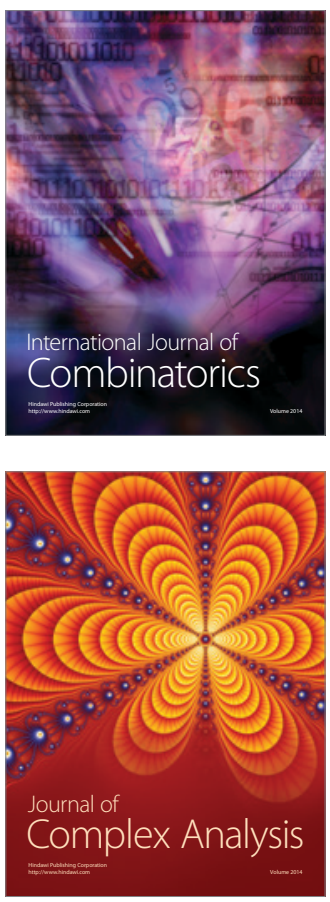

International Journal of

Mathematics and

Mathematical

Sciences
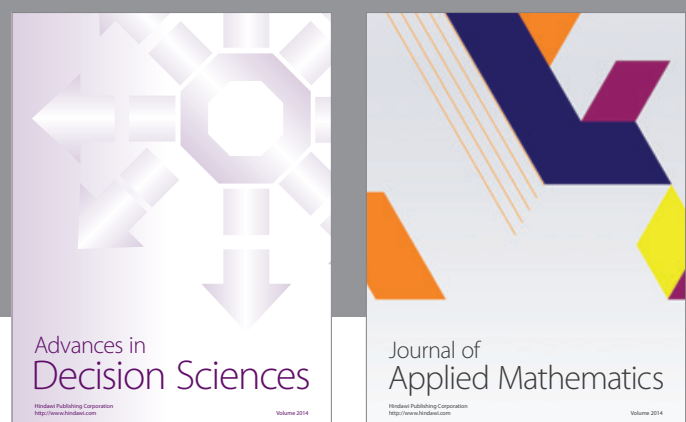

Journal of

Applied Mathematics
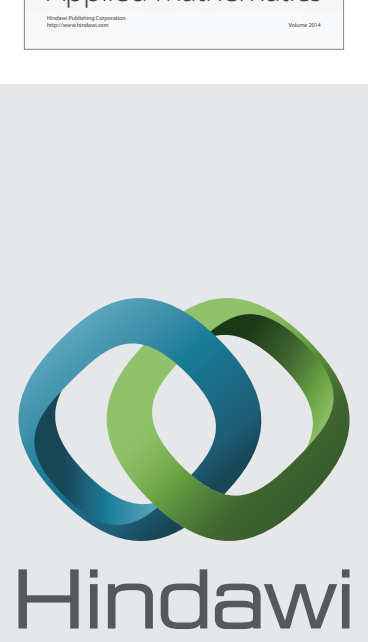

Submit your manuscripts at http://www.hindawi.com
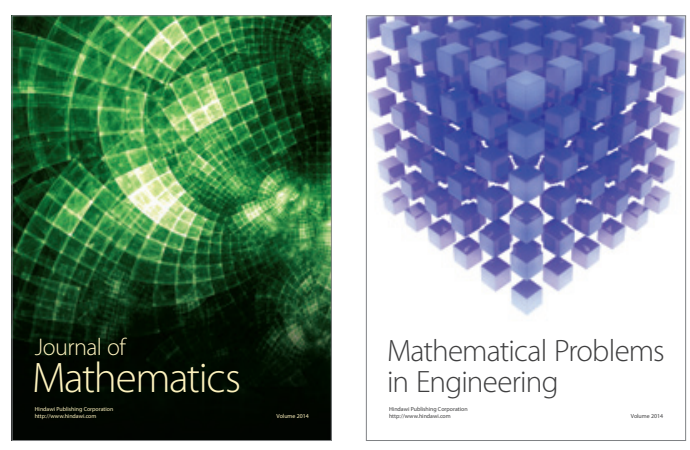

Mathematical Problems in Engineering
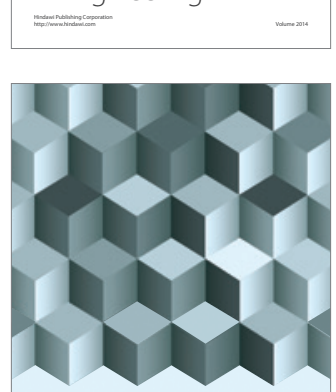

Journal of

Function Spaces
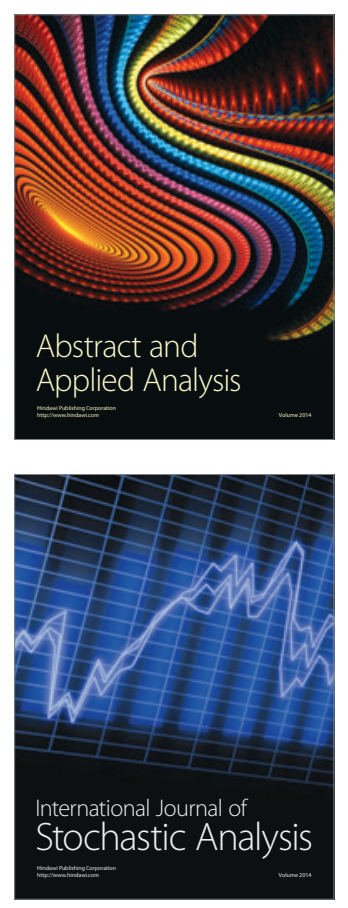

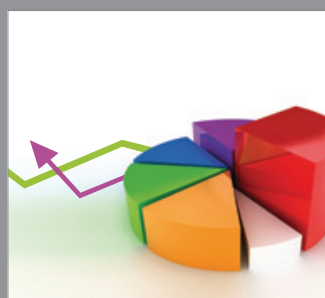

ournal of

Probability and Statistics

Promensencen
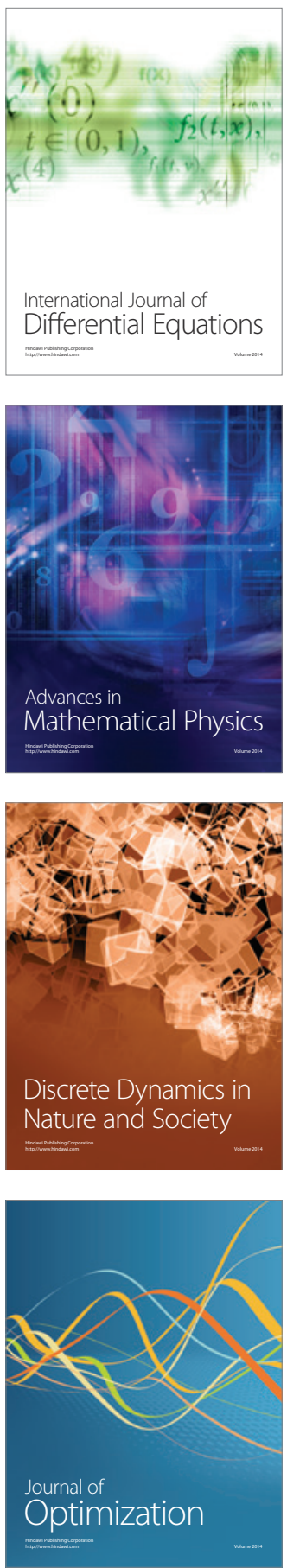